\title{
Emerging role of Alzheimer's disease-associated ubiquilin-1 in protein aggregation
}

\author{
Annakaisa Haapasalo*, Jayashree Viswanathan*, Lars Bertram†‡, Hilkka Soininen*, Rudolph E. Tanził and Mikko \\ Hiltunen:1 \\ "Department of Neurology, University of Kuopio, and Kuopio University Hospital, Kuopio, Finland, †Department of Vertebrate Genomics, Max-Planck-Institute \\ for Molecular Genetics, Berlin, Germany, and ¥Genetics and Aging Research Unit, Massachusetts General Hospital/Harvard Medical School, Charlestown, MA \\ 02129, U.S.A.
}

\begin{abstract}
Abnormal protein aggregation and intracellular or extracellular accumulation of misfolded and aggregated proteins are key events in the pathogenesis of different neurodegenerative diseases. Furthermore, endoplasmic reticulum stress and impairment of the ubiquitin-proteasome system probably contribute to neurodegeneration in these diseases. A characteristic feature of AD (Alzheimer's disease) is the abnormal accumulation of $\mathrm{A} \beta$ (amyloid $\beta$-peptide) in the brain. Evidence shows that the AD-associated PS (presenilin) also forms aggregates under certain conditions and that another AD-associated protein, ubiquilin-1, controls protein aggregation and deposition of aggregated proteins. Here, we review the current knowledge of ubiquilin-1 and PS in protein aggregation and related events that potentially influence neurodegeneration.
\end{abstract}

\section{Ubiquilin-1 structure and expression}

Ubiquilin-1 [also known as PLIC-1 (protein linking IAP (integrin-associated protein) with cytoskeleton-1)] belongs to a conserved protein family containing (i) an $\mathrm{N}$-terminal UBL domain (ubiquitin-like domain), which mediates ubiquilin interaction with the ubiquitin-interacting motif of the S5a component of the 19S subunit of the proteasome, and (ii) a C-terminal UBA domain (ubiquitin-associated domain), which preferentially binds poly-ubiquitinated proteins. These features suggest that ubiquilin-1 regulates proteasomal degradation of proteins by connecting the ubiquitination machinery and the proteasome [1-3]. Ubiquilin-1 also contains distinctive conserved asparagine- and proline-rich repeats that may mediate ubiquilin interaction with specific protein domains, such as the EH [EPS15 (epidermal growth factor substrate 15) homology] domain found in many proteins involved in endocytosis and vesicle sorting [4-6].

Ubiquilin-1 mRNA and protein are ubiquitously expressed in different tissues, such as skeletal muscle, heart, brain, kidney and liver, and different sizes of the mRNAs detected suggest the existence of different splice forms. Indeed, we have identified four different ubiquilin1 alternatively spliced TVs (transcript variants) (TV1-4)

Key words: aggresome, Alzheimer's disease (AD), presenilin, proteasome, protein aggregation, ubiquilin-1.

Abbreviations used: A $\beta$, amyloid $\beta$-peptide; AD, Alzheimer's disease; APP, amyloid precurso protein; CHOP, C/EBP (CCAAT/enhancer-binding protein)-homologous protein; CTF, C-termina fragment; EPS15, epidermal growth factor substrate 15; ER, endoplasmic reticulum; ERAD, ERassociated degradation; HD, Huntington's disease; HERP, homocysteine-induced ER protein; HMM, high-molecular-mass; IAP, integrin-associated protein; MTOR, mammalian target of rapamycin; $n A C h R$, nicotinic acetylcholine receptor; NFT, neurofibrillary tangle; PS, presenilin; PS $/ \gamma$ secretase, PS-dependent $\gamma$-secretase; PSEN, PS gene; SNP, single nucleotide polymorphism; TDP-43, 43 kDa TAR DNA-binding protein; TV, transcript variant; UBA domain, ubiquitin-associated domain; UBL domain, ubiquitin-like domain; UBQLN1, ubiquilin-1 gene; UPR, unfolded protein response; UPS, ubiquitin-proteasome system.

${ }^{1}$ To whom correspondence should be addressed (e-mail mikko.hiltunen@uku.fi). from human brain $[7,8]$. Staining of cells with ubiquilin-1 antibodies has indicated cytoplasmic subcellular localization, with some immunoreactivity at the periphery of the cells [6]. Furthermore, staining of human brain revealed a prominent presence of ubiquilin-1 in neurons [5].

\section{Ubiquilin-1-interacting proteins}

A yeast two-hybrid screen demonstrated that ubiquilin-1 interacts with the $\mathrm{AD}$ (Alzheimer's disease)-associated PS (presenilin) [5] (see below). Similarly, ubiquilin-1 was shown to interact with mTOR (mammalian target of rapamycin), a major inhibitor of autophagocytosis, but the function of this interaction is unclear [6]. Ubiquilin-1 also binds other proteins, such as bHLH (basic helixloop-helix) transcription factors [9] and IAP CD47 to induce cell spreading [10] and heterotrimeric G-protein to inhibit cell migration [11]. Furthermore, ubiquilin-1, enriched at inhibitory synapses, enhances membrane insertion, cell surface number and subunit stability of GABA ( $\gamma$-aminobutyric acid) receptors and thus may regulate synaptic inhibition [12,13]. Finally, ubiquilin-1 regulates nicotine-induced up-regulation of neuronal $\mathrm{nAChR}$ (nicotinic acetylcholine receptors) by directing unassembled $\mathrm{nAChR}$ subunits for proteasomal degradation and therefore limiting nAChR levels on the plasma membrane [14]. Mounting evidence suggests that ubiquilin-1 regulates the levels and UPS (ubiquitin-proteasome system)-mediated degradation of several other proteins as well (see below).

\section{Ubiquilin-1 is an AD-associated protein}

$\mathrm{AD}$ is the most common cause of dementia, characterized by global cognitive decline and memory impairment 
[15]. Neuropathological hallmarks of $\mathrm{AD}$ brain include extracellular amyloid plaques, consisting of $\mathrm{A} \beta$ (amyloid $\beta$-peptide), intraneuronal NFTs (neurofibrillary tangles), composed of the hyperphosphorylated MAP (microtubuleassociated protein) tau protein, and a diffuse loss of neurons and synapses in the cortex [16]. $\mathrm{A} \beta$ is proteolytically produced from its precursor APP (amyloid precursor protein) by BACE ( $\beta$-site APP-cleaving enzyme) and $\mathrm{PS} / \gamma$ secretase (PS-dependent $\gamma$-secretase) [17].

We have demonstrated that SNPs (single nucleotide polymorphisms) in the UBQLN1 (ubiquilin-1 gene), genetically associate with $\mathrm{AD}$ [8]. The risk-conferring haplotype was defined by a single intronic SNP downstream of exon 8 (rs12344615, also known as 'intron 8'). Furthermore, the risk allele was associated with an increased ratio of ubiquilin1 TV2 to the full-length TV1 in AD brain. These results suggest that genetic variants of $U B Q L N 1$ increase the risk of $\mathrm{AD}$, possibly by affecting the alternative splicing of the $U B Q L N 1$ gene [8]. Although genetic association between $U B Q L N 1$ and $\mathrm{AD}$ was not found in all replicate studies in different populations [18-24] (for an up-to-date overview of $\mathrm{AD}$ genetic association studies on $U B Q L N 1$, see the AlzGene website at http://www.alzgene.org; [25]), some studies have confirmed that $U B Q L N 1$ is associated with risk for $\mathrm{AD}$ $[18,22,24]$.

As further substantiation of the link between ubiquilin-1 and $\mathrm{AD}$, we found that ubiquilin-1 interacts with APP and that knockdown of ubiquilin-1 in human neuroglioma cells increases APP maturation, trafficking to plasma membrane and proteolytic processing, as shown by augmented levels of APP CTFs (C-terminal fragments) and secretion of soluble $\mathrm{N}$-terminal APP peptides and $\mathrm{A} \beta$. Therefore we propose that ubiquilin-1 controls APP trafficking to the plasma membrane through the secretory pathway and thus influences $\mathrm{A} \beta$ generation [26]. Agreeing with these results, co-overexpression of the Drosophila ubiquilin orthologue $d U b q \ln$ with $h A P P$ (human APP) in the fly eye decreased full-length APP and APP CTF levels in vivo. Moreover, silencing of $d U b q l n$ in the central nervous system in Drosophila caused age-dependent neurodegeneration and shortened life span. In the eye, silencing of $d U b q \ln$ enhanced retinal degeneration induced by $d P s n$ (Drosophila PS) overexpression, whereas overexpression of $d U b q \ln$ rescued the $d P s n$-induced degeneration and eye malformation, suggesting that ubiquilin-1 is cytoprotective [27]. In contrast to our findings, Zhang et al. [28] showed that ubiquilin-1 knockdown in human neuroblastoma cells decreased APP maturation and processing. Also, ubiquilin-1 knockdown in transgenic flies was shown to decrease the steadystate levels of APP and APP CTF, whereas ubiquilin-1 overexpression had an opposite effect [29]. Even though these studies show contrasting results to our previous results, they undoubtedly demonstrate that ubiquilin-1 affects maturation and processing of APP, perhaps in a cell-type-specific manner and thus it may affect $\mathrm{AD}$ pathogenesis.

Ubiquilin antibodies co-stain NFTs in the AD brain and Lewy body inclusions in the brain of individuals with PD
(Parkinson's disease) [5]. Also ubiquitin immunoreactivity is strongly associated with NFTs and $\mathrm{A} \beta$ plaques in $\mathrm{AD}$ brain $[30,31]$. Moreover, ubiquilin-1 associates with intranuclear inclusions containing expanded polyglutamine protein in the cell and the mouse model of HD (Huntington's disease) [32]. Ubiquilin overexpression suppressed the aggregation and toxicity of polyglutamine and polyalanine proteins in cells and a Caenorbabditis elegans model of HD $[33,34]$. Furthermore, ubiquilin interacted with and enhanced the degradation of expanded polyglutamine proteins, possibly by targeting them to proteasomal degradation [35]. Collectively, these findings support the notion that ubiquilin-1 plays an important role in the pathogenesis of $\mathrm{AD}$ and also other slowly progressing neurodegenerative diseases involving abnormal protein aggregation.

\section{Ubiquilin-1 and cytoprotection under stress conditions}

ER (endoplasmic reticulum) stress is activated in neurodegeneration and PSEN (PS gene) mutations impair UPR (unfolded protein response), implying that these events are affected in AD [36,37]. UPR is activated when cells undergo stress and unfolded proteins accumulate in the ER. To overcome ER stress, ER-resident molecular chaperones and foldases are activated, protein synthesis is down-regulated and clearance of unfolded proteins through ERAD (ERassociated degradation) is enhanced in cells. A report by Ko et al. [38] indicated that under hypoxic conditions, the levels of ubiquilin-1 and protein disulfide-isomerase, a foldase, were up-regulated and ubiquilin-1 attenuated the induction of $\mathrm{CHOP}[\mathrm{C} / \mathrm{EBP}$ (CCAAT/enhancer-binding protein)homologous protein], a UPR-inducible transcription factor. Similarly, we showed recently that, under tunicamycininduced ER stress, ubiquilin-1 TVs TV1, TV2 and TV3 attenuated $\mathrm{CHOP}$ induction and increased cell viability [7]. Interestingly, TV3, which lacks most of the UBL domain, had the strongest cytoprotective effect among the variants [7]. Furthermore, ubiquilins, recruited by HERP (homocysteine-induced ER protein), a protein upregulated in response to ER stress, are involved in ERADdependent degradation of HERP-dependent substrates [39]. In summary, these observations suggest that ubiquilin-1 plays a role in the UPR and confers cytoprotection under stress.

\section{Ubiquilin-1 interacts with AD-associated PS}

PS plays a fundamental role in $\mathrm{AD}$ pathogenesis. First, mutations in the PSEN1 and PSEN2 genes encoding PS1 and PS2 respectively cause early-onset $A D$ and increase $\mathrm{A} \beta$ generation $[40,41]$. Secondly, PS is an essential catalytic subunit of the PS $/ \gamma$-secretase complex, cleaving APP to generate $\mathrm{A} \beta$ [16]. Ubiquilin was identified as a PS-interacting protein in a yeast two-hybrid screen and was shown to 
directly bind to and co-localize with PS1 and PS2 in the same subcellular compartments [5]. Our studies by using fluorescence resonance energy-based techniques confirmed these findings and indicated that the co-localization and interaction of ubiquilin-1 and PS1 take place near the cell surface in cells and mouse primary neurons. Furthermore, we showed that PS1 and ubiquilin-1 interact in human brain in both control and $\mathrm{AD}$ subjects [42].

Previous studies indicate that PSs undergo ubiquitination and proteasomal degradation [43-45]. Ubiquilin was demonstrated to increase accumulation of PS, but not various other proteins, to form ubiquitinated HMM (highmolecular-mass) complexes, suggesting that it specifically promotes PS aggregation [5]. In line with this, ubiquilin-1 overexpression increased the levels of full-length PS1 and PS2 and selectively reduced the proteasomal degradation of HMM PS2. Moreover, ubiquilin-1 co-localized with ubiquitinpositive structures and aggresomes in cells, indicating association with ubiquitinated proteins. It is unclear, however, whether the stabilization of PS2 and HMM PS2 resulted from the fact that ubiquilin-1 prevented polyubiquitin chain elongation and thus recognition by the proteasome, or whether ubiquilin-1 by binding to the polyubiquitin chain on PS2 physically prevented its interaction with the proteasome [46]. In addition, overexpression of ubiquilin-1 or -2 was reported to decrease the levels of PS N-terminal fragment or PS CTF and two other PS $/ \gamma$-secretase complex components, nicastrin and Pen-2 (presenilin enhancer-2 subunit of $\gamma$ secretase), whereas ubiquilin knockdown had an opposite effect [47]. These results suggest that ubiquilin regulates PS endoproteolysis and PS $/ \gamma$-secretase complex component levels or, alternatively, that PS endoproteolysis may be prevented because full-length PS aggregates in the presence of ubiquilin. On the other hand, we did not observe any effects of ubiquilin-1 knockdown on the steady-state levels of the complex components or activity of $\mathrm{PS} / \gamma$-secretase in different cells [26]. Thus, although clarifying the impact of ubiquilin1 on $\mathrm{PS} / \gamma$-secretase requires further studies, these findings together emphasize that ubiquilin-1 regulates PS degradation and aggregation.

\section{Aggresomes and PS}

As ubiquilin interacts with PS, increases HMM PS formation, associates with polyubiquitinated proteins and was shown to co-localize with PS2 in the aggresomes, it is possible that ubiquilin targets aggregated PS to the aggresomes $[5,46]$. Accumulation of misfolded or aggregated proteins is a characteristic of many neurodegenerative diseases. In response to protein misfolding, molecular chaperones, foldases and the UPS are activated to refold or discard the potentially toxic aggregates. However, when this machinery becomes overwhelmed, the cytoplasmic protein aggregates are actively transported along the microtubules to a perinuclear location to form inclusion bodies termed aggresomes. Aggresomes are composed of molecular chaperones and proteasomal subunits, in addition to aggregated potentially polyubiquitinated proteins, and they co-localize with centrosomal proteins, such as $\gamma$ tubulin. Furthermore, typical of aggresomes is redistribution of the intermediate filament protein vimentin to form a cage surrounding the aggresomal core, which may contribute to the stability of the aggresomes [48]. Autophagosomes and lysosomes have been found to accumulate around the aggresomes, suggesting that aggresomes may be disposed of by autophagocytosis. Therefore aggresome formation is considered as a protective phenomenon to remove aggregated proteins from the cytosol, where they might interfere with cellular function, and an intermediate step before autophagic degradation. The aggresome-autophagy pathway and its relation to neurodegenerative diseases are discussed elsewhere $[48,49]$.

Studies by Johnston et al. [50] using CFTR (cystic fibrosis transmembrane conductance regulator), an inefficiently folded membrane protein undergoing UPS-dependent degradation, as a model protein have been central to the characterization of aggresome formation. These authors also showed that degradation of PS1 and PS1 containing a familial $\mathrm{AD}$ mutation (PS1/A246E) was prevented under proteasomal inhibition. This resulted in the accumulation of detergentinsoluble full-length and HMM forms of PS1 and PS1/A246E and redistribution of the aggregated PSs into aggresomes that were surrounded by a vimentin envelope [50]. Another report indicated that also a novel PS-binding protein, specifically expressed in the brain, was present in aggresomes in cells and primary neurons [51]. Whether ubiquilin-1 directly regulates PS targeting to the aggresomes and whether this has implications in $\mathrm{AD}$ pathogenesis remain interesting open questions. Figure 1 shows the possible role of ubiquilin- 1 in PS targeting to the proteasome or aggresome.

\section{Ubiquilin-1, protein aggregation and aggresome formation}

Evidence in a polyglutamine disease model shows that ubiquilin-1 and EPS15 (an endocytic protein) localized to the aggresomes and that polyglutamine enhanced their in vivo interaction [52]. Moreover, knockdown of ubiquilin-1 and EPS15 decreased aggresome formation, and the UBL domain of ubiquilin-1 was proven to be prerequisite for polyglutamine transport to the aggresomes and association of EPS15 with the aggregates [52]. These results propose that during increased protein aggregation when the proteasome becomes overwhelmed, the interaction of ubiquilin-1 with EPS15 is enhanced, promoting deposition of aggregated proteins to aggresomes [52]. Furthermore, it was shown recently that ubiquilins regulate autophagydependent cell survival under nutrient starvation [53]. Overexpression of ubiquilin-1 or -2 protected cells from death, whereas knockdown of ubiquilins rendered the cells susceptible during starvation. Ubiquilins were demonstrated to associate with autophagosomes through their UBA domain, and this association was required to mediate 
Figure 1 Model for the possible role of ubiquilin-1 in targeting PS to the proteasome or aggresome

The ubiquilin N-terminus interacts with multiple sites in PS [41]. Ubiquilin-1 contains an N-terminal UBL domain and a C-terminal UBA domain. It also contains asparagine- and proline-rich repeats (Asn-Pro) that may mediate protein-protein interactions [5]. During PS misfolding (1a), ubiquilin-1 binds to the polyubiquitin (Ub) chain on PS via its UBA domain and brings PS to the proteasome for degradation. Ubiquilin-1-proteasome interaction is mediated by the UBL domain [2]. During PS aggregation (1b), polyubiquitinated PSS accumulate and form HMM complexes, which cannot be degraded by the proteasome. Ubiquilin- 1 binds to the polyubiquitinated HMM PS and targets it to the aggresome [5,50] (2). The cytosolic HMM PS complexes are transported along microtubules on retrograde motors to a perinuclear location, where they co-localize with centrosomal $\gamma$-tubulin (grey dots) in the nuclear indentation (3). The aggresome containing HMM PS is surrounded by vimentin envelope [48] (4). Subsequently, aggresomes may be degraded by autophagocytosis. Ubiquilin-1 may also target other aggregated proteins to the aggresome-autophagosome pathway in a similar manner.

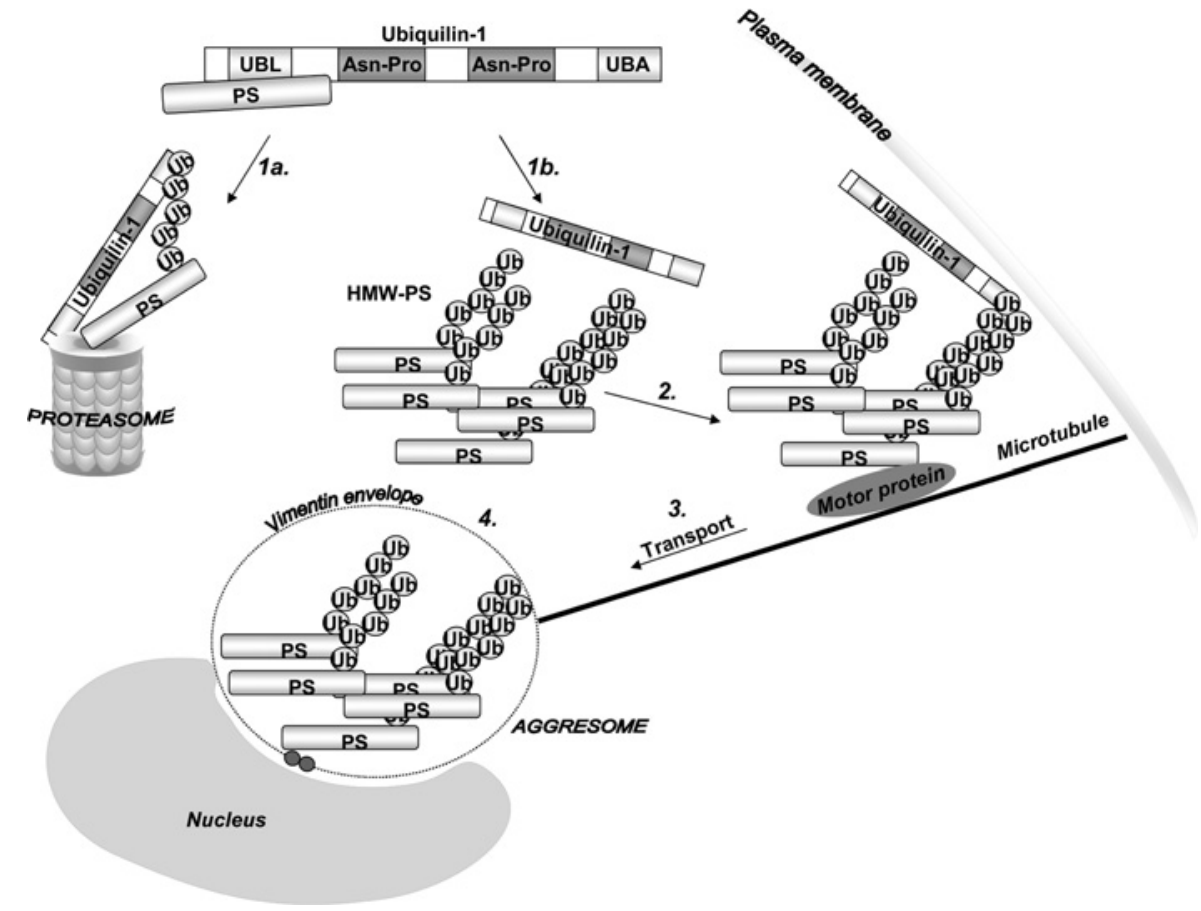

the cytoprotection. Additionally, ubiquilin associated with autophagy-destined protein aggregates, including aggregated huntingtin, an $\mathrm{HD}$-associated protein [53]. It is also worth noting that ubiquilin-1 was previously shown to interact with mTOR, a key inhibitor of autophagy, and that inhibition of mTOR induced autophagy and reduced the toxicity of polyglutamine expansions in HD models $[6,54]$.

Recently, ubiquilin-1 was proven to interact via its UBA domain with polyubiquitinated TDP-43 (43 kDa TAR DNA-binding protein), a protein present in ubiquitinpositive cytoplasmic neuronal aggregates in subjects with frontotemporal dementia and amyotrophic lateral sclerosis [53]. Dependent on its UBA domain, ubiquilin-1 stimulated the aggregation and targeting of TDP-43 to autophagosomes. Interestingly, the ubiquilin-1 allelic variant lacking exon 8 , which we found to be genetically associated with AD risk, failed to efficiently recruit TDP-43 to the cytosolic aggregates [55], suggesting that the lack of exon 8 may have functional correlates beyond those described above.
Taken together, ubiquilin-1 appears to play a central role in the regulation of protein aggregation and targeting of aggregated proteins to the aggresome-autophagosome pathway for disposal under conditions of excessive protein aggregation related to different neurodegenerative diseases.

\section{Conclusions}

The findings in different cell lines in vitro and disease models in vivo indicate an important role for ubiquilin-1 in the targeting of different proteins for degradation to the UPS under normal conditions or to the aggresomeautophagosome pathway under conditions when aggregated proteins accumulate excessively. Therefore ubiquilin-1 appears to function as a cytoprotective protein not only in $\mathrm{AD}$ pathogenesis, but also in that of other neurodegenerative diseases involving abnormal protein aggregation, ER stress and potential impairment of the UPS. Future studies will elucidate in more detail how ubiquilin-1 exerts its function to 
regulate the delicate balance between protein degradation and aggregation.

\section{Funding}

This work was supported by the Nordic Centre of Excellence in Neurodegeneration; Academy of Finland; BiND (Biology of Neurodegenerative Diseases) Marie Curie EST (Early Stage Researcher Training) Programme; and Kuopio University Hospital [EVO grant 5772208].

\section{References}

1 Kleijnen, M.F., Shih, A.H., Zhou, P., Kumar, S., Soccio, R.E., Kedersha, N.L. Gill, G. and Howley, P.M. (2000) The hPLIC proteins may provide a link between the ubiquitination machinery and the proteasome. Mol. Cell $\mathbf{6}$ 409-419

2 Kleijnen, M.F., Alarcon, R.M. and Howley, P.M. (2003) The ubiquitin-associated domain of hPLIC-2 interacts with the proteasome. Mol. Biol. Cell 14, 3868-3875.

3 Ko, H.S., Uehara, T., Tsuruma, K. and Nomura, Y. (2004) Ubiquilin interacts with ubiquitylated proteins and proteasome through its ubiquitin-associated and ubiquitin-like domains. FEBS Lett. 566, 110-114

4 Mayer, B.J. (1999) Endocytosis: EH domains lend a hand. Curr. Biol. 9, R70-R73

5 Mah, A.L., Perry, G., Smith, M.A. and Monteiro, M.J. (2000) Identification of ubiquilin, a novel presenilin interactor that increases presenilin protein accumulation. J. Cell Biol. 151, 847-862

6 Wu, S., Mikhailov, A., Kallo-Hosein, H., Hara, K., Yonezawa, K. and Avruch, J. (2002) Characterization of ubiquilin 1, an mTOR-interacting protein. Biochim. Biophys. Acta 1542, 41-56

7 Lu, A., Hiltunen, M., Romano, D.M., Soininen, H., Hyman, B.T., Bertram, L. and Tanzi, R.E. (2009) Effects of ubiquilin 1 on the unfolded protein response. J. Mol. Neurosci. 38, 19-30

8 Bertram, L., Hiltunen, M., Parkinson, M., Ingelsson, M., Lange, C., Ramasamy, K., Mullin, K., Menon, R., Sampson, A.J., Hsiao, M.Y. et al. (2005) Family-based association between Alzheimer's disease and variants in UBOLN1. N. Engl. J. Med. 352, 884-894

9 Persson, P., Stockhausen, M.T., Pahlman, S. and Axelson, H. (2004) Ubiquilin-1 is a novel HASH-1-complexing protein that regulates levels of neuronal bHLH transcription factors in human neuroblastoma cells. Int. J. Oncol. 25, 1213-1221

10 Wu, A.L., Wang, J., Zheleznyak, A. and Brown, E.J. (1999) Ubiquitin-related proteins regulate interaction of vimentin intermediate filaments with the plasma membrane. Mol. Cell 4, 619-625

11 N'Diaye, E.N. and Brown, E.J. (2003) The ubiquitin-related protein PLIC-1 regulates heterotrimeric $\mathrm{G}$ protein function through association with $\mathrm{G} \beta \gamma$. J. Cell Biol. 163, 1157-1165

12 Bedford, F.K., Kittler, J.T., Muller, E., Thomas, P., Uren, J.M., Merlo, D. Wisden, W., Triller, A., Smart, T.G. and Moss, S.J. (2001) GABA receptor cell surface number and subunit stability are regulated by the ubiquitin-like protein plic-1. Nat. Neurosci. 4, 908-916

13 Saliba, R.S., Pangalos, M. and Moss, S.J. (2008) The ubiquitin-like protein plic-1 enhances the membrane insertion of $\mathrm{GABA}_{\mathrm{A}}$ receptors by increasing their stability within the endoplasmic reticulum. J. Biol. Chem. 283, 18538-18544

14 Ficklin, M.B., Zhao, S. and Feng, G. (2005) Ubiquilin-1 regulates nicotine-induced up-regulation of neuronal nicotinic acetylcholine receptors. J. Biol. Chem. 280, 34088-34095

15 Tanzi, R.E. and Bertram, L. (2005) Twenty years of the Alzheimer's disease amyloid hypothesis: a genetic perspective. Cell 120, 545-555

16 Selkoe, D.J. (2004) Cell biology of protein misfolding: the examples of Alzheimer's and Parkinson's diseases. Nat. Cell Biol. 6, 1054-1061

17 Vetrivel, K.S. and Thinakaran, G. (2006) Amyloidogenic processing of $\beta$-amyloid precursor protein in intracellular compartments. Neurology 66, S69-S73

18 Slifer, M.A., Martin, E.R., Haines, J.L. and Pericak-Vance, M.A. (2005) The ubiquilin 1 gene and Alzheimer's disease. N. Engl. J. Med. 352, 2752-2753
19 Smemo, S., Nowotny, P., Hinrichs, A.L., Kauwe, J.S., Cherny, S., Erickson, K., Myers, A.J., Kaleem, M., Marlowe, L., Gibson, A.M. et al. (2006) Ubiquilin 1 polymorphisms are not associated with late-onset Alzheimer's disease. Ann. Neurol. 59, 21-26

20 Bensemain, F., Chapuis, J., Tian, J., Shi, J., Thaker, U., Lendon, C., Iwatsubo, T., Amouyel, P., Mann, D. and Lambert, J.C. (2006) Association study of the ubiquilin gene with Alzheimer's disease. Neurobiol. Dis. 22, 691-693

21 Brouwers, N., Sleegers, K., Engelborghs, S., Bogaerts, V., van Duijn, C.M., De Deyn, P.P., Van Broeckhoven, C. and Dermaut, B. (2006) The UBQLN1 polymorphism, UBQ-8i, at 9q22 is not associated with Alzheimer's disease with onset before 70 years. Neurosci. Lett. 392, 72-74

22 Kamboh, M.I., Minster, R.L., Feingold, E. and DeKosky, S.T. (2006) Genetic association of ubiquilin with Alzheimer's disease and related quantitative measures. Mol. Psychiatry 11, 273-279

23 Arias-Vasquez, A., de Lau, L., Pardo, L., Liu, F., Feng, B.J., Bertoli-Avella, A., Isaacs, A., Aulchenko, Y., Hofman, A., Oostra, B. et al. (2007) Relationship of the ubiquilin 1 gene with Alzheimer's and Parkinson's disease and cognitive function. Neurosci. Lett. 424, 1-5

24 Golan, M.P., Melquist, S., Safranow, K., Styczynska, M., Slowik, A., Kobrys, M., Zekanowski, C. and Barcikowska, M. (2008) Analysis of UBQLN1 variants in a polish Alzheimer's disease patient: control series. Dement. Geriatr. Cogn. Disord. 25, 366-371

25 Bertram, L., McQueen, M.B., Mullin, K., Blacker, D. and Tanzi, R.E. (2007) Systematic meta-analyses of Alzheimer disease genetic association studies: the AlzGene database. Nat. Genet. 39, 17-23

26 Hiltunen, M., Lu, A., Thomas, A.V., Romano, D.M., Kim, M., Jones, P.B., Xie, Z., Kounnas, M.Z., Wagner, S.L., Berezovska, 0. et al. (2006) Ubiquilin 1 modulates amyloid precursor protein trafficking and $A \beta$ secretion. J. Biol. Chem. 281, 32240-32253

27 Li, A., Xie, Z., Dong, Y., McKay, K.M., McKee, M.L. and Tanzi, R.E. (2007) Isolation and characterization of the Drosophila ubiquilin ortholog dubqln: in vivo interaction with early-onset Alzheimer disease genes. Hum. Mol. Genet. 16, 2626-2639

28 Zhang, C., Khandelwal, P.J., Chakraborty, R., Cuellar, T.L., Sarangi, S. Patel, S.A., Cosentino, C.P., O'Connor, M., Lee, J.C., Tanzi, R.E. and Saunders, A.). (2007) An AICD-based functional screen to identify APP metabolism regulators. Mol. Neurodegener. 2, 15

29 Gross, G.G., Feldman, R.M., Ganguly, A., Wang, J., Yu, H. and Guo, M. (2008) Role of X11 and ubiquilin as in vivo regulators of the amyloid precursor protein in Drosophilo. PLoS ONE 3, e2495

30 Mori, H., Kondo, J. and Ihara, Y. (1987) Ubiquitin is a component of paired helical filaments in Alzheimer's disease. Science 235, 1641-1644

31 Perry, G., Friedman, R., Shaw, G. and Chau, V. (1987) Ubiquitin is detected in neurofibrillary tangles and senile plaque neurites of Alzheimer disease brains. Proc. Natl. Acad. Sci. U.S.A. 84, 3033-3036

32 Doi, H., Mitsui, K., Kurosawa, M., Machida, Y., Kuroiwa, Y. and Nukina, N. (2004) Identification of ubiquitin-interacting proteins in purified polyglutamine aggregates. FEBS Lett. 571, 171-176

33 Wang, H., Lim, P.J., Yin, C., Rieckher, M., Vogel, B.E. and Monteiro, M.J. (2006) Suppression of polyglutamine-induced toxicity in cell and animal models of Huntington's disease by ubiquilin. Hum. Mol. Genet. 15, 1025-1041

34 Wang, H. and Monteiro, M.J. (2007) Ubiquilin overexpression reduces GFP-polyalanine-induced protein aggregates and toxicity. Exp. Cell Res. 313, 2810-2820

35 Wang, H. and Monteiro, M.J. (2007) Ubiquilin interacts and enhances the degradation of expanded-polyglutamine proteins. Biochem. Biophys. Res. Commun. 360, 423-427

36 Katayama, T., Imaizumi, K., Sato, N., Miyoshi, K., Kudo, T., Hitomi, J. Morihara, T., Yoneda, T., Gomi, F., Mori, Y. et al. (1999) Presenilin-1 mutations downregulate the signalling pathway of the unfolded-protein response. Nat. Cell Biol. 1, 479-485

37 Uehara, T., Nakamura, T., Yao, D., Shi, Z.Q., Gu, Z., Ma, Y., Masliah, E., Nomura, Y. and Lipton, S.A. (2006) S-nitrosylated protein-disulphide isomerase links protein misfolding to neurodegeneration. Nature $\mathbf{4 4 1}$, 513-517

38 Ko, H.S., Uehara, T. and Nomura, Y. (2002) Role of ubiquilin associated with protein-disulfide isomerase in the endoplasmic reticulum in stress-induced apoptotic cell death. J. Biol. Chem. 277, 35386-35392

39 Kim, T.Y., Kim, E., Yoon, S.K. and Yoon, J.B. (2008) Herp enhances ER-associated protein degradation by recruiting ubiquilins. Biochem. Biophys. Res. Commun. 369, 741-746

40 Sherrington, R., Rogaev, E.I., Liang, Y., Rogaeva, E.A., Levesque, G., Ikeda, M., Chi, H., Lin, C., Li, G. and Holman, K. (1995) Cloning of a gene bearing missense mutations in early-onset familial Alzheimer's disease. Nature 375, 754-760 
41 Levy-Lahad, E., Wasco, W., Poorkaj, P., Romano, D.M., Oshima, J., Pettingell, W.H., YU, C.E., Jondro, P.D., Schmidt, S.D. and Wang, K. (1995) Candidate gene for the chromosome 1 familial Alzheimer's disease locus. Science 269, 973-977

42 Thomas, A.V., Herl, L., Spoelgen, R., Hiltunen, M., Jones, P.B., Tanzi, R.E., Hyman, B.T. and Berezovska, O. (2006) Interaction between presenilin 1 and ubiquilin 1 as detected by fluorescence lifetime imaging microscopy and a high-throughput fluorescent plate reader. J. Biol. Chem. 281 26400-26407

43 Kim, T.W., Pettingell, W.H., Hallmark, O.G., Moir, R.D., Wasco, W. and Tanzi, R.E. (1997) Endoproteolytic cleavage and proteasomal degradation of presenilin 2 in transfected cells. J. Biol. Chem. 272 11006-11010

44 Fraser, P.E., Levesque, G., Yu, G., Mills, L.R., Thirlwell, J., Frantseva, M., Gandy, S.E., Seeger, M., Carlen, P.L. and St George-Hyslop, P. (1998) Presenilin 1 is actively degraded by the 265 proteasome. Neurobiol. Aging 19, S19-S21

45 Honda, T., Yasutake, K., Nihonmatsu, N., Mercken, M., Takahashi, H., Murayama, O., Murayama, M., Sato, K., Omori, A., Tsubuki, S. et al. (1999) Dual roles of proteasome in the metabolism of presenilin 1. J. Neurochem. 72, 255-261

46 Massey, L.K., Mah, A.L., Ford, D.L., Miller, J., Liang, J., Doong, H. and Monteiro, M.J. (2004) Overexpression of ubiquilin decreases ubiquitination and degradation of presenilin proteins. J. Alzheimers Dis. 6, 79-92

47 Massey, L.K., Mah, A.L. and Monteiro, M.J. (2005) Ubiquilin regulates presenilin endoproteolysis and modulates $\gamma$-secretase components, pen-2 and nicastrin. Biochem. J. 391, 513-525
48 Kopito, R.R. (2000) Aggresomes, inclusion bodies and protein aggregation. Trends Cell Biol. 10, 524-530

49 olzmann, J.A., Li, L. and Chin, L.S. (2008) Aggresome formation and neurodegenerative diseases: therapeutic implications. Curr. Med. Chem. 15, $47-60$

50 Johnston, J.A., Ward, C.L. and Kopito, R.R. (1998) Aggresomes: a cellular response to misfolded proteins. J. Cell Biol. 143, 1883-1898

51 Namekata, K., Nishimura, N. and Kimura, H. (2002) Presenilin-binding protein forms aggresomes in monkey kidney cos-7 cells. J. Neurochem. 82, 819-827

52 Heir, R., Ablasou, C., Dumontier, E., Elliott, M., Fagotto-Kaufmann, C. and Bedford, F.K. (2006) The UBL domain of PLIC-1 regulates aggresome formation. EMBO Rep. 7, 1252-1258

53 N'Diaye, E.N., Kajihara, K.K., Hsieh, I., Morisaki, H., Debnath, J. and Brown, E.J. (2009) PLIC proteins or ubiquilins regulate autophagy-dependent cell survival during nutrient starvation. EMBO Rep. 10, 173-179

54 Ravikumar, B., Vacher, C., Berger, Z., Davies, J.E., Luo, S., Oroz, L.G., Scaravilli, F., Easton, D.F., Duden, R., O'Kane, C.J. and Rubinsztein, D.C. (2004) Inhibition of mTOR induces autophagy and reduces toxicity of polyglutamine expansions in fly and mouse models of Huntington disease. Nat. Genet. 36, 585-595

55 Kim, S.H., Shi, Y., Hanson, K.A., Williams, L.M., Sakasai, R., Bowler, M.J. and Tibbetts, R.S. (2009) Potentiation of amyotrophic lateral sclerosis (ALS)-associated TDP-43 aggregation by the proteasome-targeting factor, ubiquilin 1. J. Biol. Chem. 284, 8083-8092

Received 1 July 2009

doi:10.1042/BST0380150 